\title{
Concentration dependence of Förster resonant energy transfer between donor and acceptor nanocrystal quantum dot layers: Effect of donor-donor interactions
}

\author{
Manuela Lunz and A. Louise Bradley* \\ Semiconductor Photonics Group, School of Physics, Trinity College Dublin, Dublin 2, Ireland
}

Valerie A. Gerard, Stephen J. Byrne, and Yurii K. Gun'ko

School of Chemistry, Trinity College Dublin, Dublin 2, Ireland

Vladimir Lesnyak and Nikolai Gaponik

Physical Chemistry, TU Dresden, Bergstrasse 66b, D-01062 Dresden, Germany

(Received 9 August 2010; revised manuscript received 23 December 2010; published 11 March 2011)

\begin{abstract}
The influences of donor and acceptor concentrations on Förster resonant energy transfer (FRET) in a separated donor-acceptor quantum dot bilayer structure have been investigated. Donor intra-ensemble energy transfer is shown to have an impact on the donor-acceptor FRET efficiency in the bilayer structure. At high donor concentrations the FRET distance dependence and the acceptor concentration dependence in the separated donor-acceptor layer structure agree well with theories developed for FRET between randomly distributed, homogeneous donor and acceptor ensembles. However, discrepancies between measurement and theory are found at low donor concentrations. A donor concentration study shows that the FRET efficiency decreases with increasing donor concentration even though a donor concentration-independent FRET efficiency is predicted by standard theory. The observed dependence of the FRET efficiency on the donor concentration can be explained within the FRET rate model, for a constant, donor concentration independent FRET rate, by taking into account the concentration dependent donor reference lifetime arising from intra-donor ensemble FRET. This shows that the decrease in the FRET efficiency with increasing donor concentration is not a signature of a change in the donor-acceptor FRET rate, but due to the competition of the donor-acceptor and donor-donor energy transfer for the higher energy donors. As the intra-donor ensemble FRET represents another decay mechanism, the donor quantum yield for the higher energy donors decreases with increasing donor quantum dot (QD) concentration, as can also be seen from the redshift of the donor emission spectrum. Using this concentration dependent donor quantum yield in the calculation of the Förster radius, the FRET theory for homogeneous donor and acceptor ensembles can be modified to include the effect of the donor intra-ensemble transfer and to correctly describe the trends and absolute values of the measured FRET efficiencies as a function of the donor and the acceptor concentrations. These results show that in QD systems where intra-donor ensemble FRET is as important as the radiative and nonradiative donor decay mechanisms, the FRET rate rather than the FRET efficiency more appropriately characterizes the donor-acceptor FRET. By fitting with the rate model, FRET rates as high as $(1.2 \mathrm{~ns})^{-1}$ have been determined for the structures presented here.
\end{abstract}

DOI: 10.1103/PhysRevB.83.115423

PACS number(s): 78.67.Hc, 78.47.jd, 81.16.Dn, 82.20.Rp

\section{INTRODUCTION}

Förster resonant energy transfer (FRET) is an energy transfer mechanism based on the interactions of donor and acceptor dipole moments. ${ }^{1-3}$ This nonradiative type of energy transfer occurs when donor and acceptor have states in resonance and if the donor-acceptor separation is short, typically only a few nanometers. ${ }^{2,3}$ The strong distance dependence of the FRET process can be used to measure distances on the nanoscale ${ }^{2,4}$ and to build sensing devices by monitoring binding events. ${ }^{5-8}$ In addition, graded energy structures can be engineered in which FRET provides a mechanism to transport energy from donor to acceptor components. ${ }^{9-12}$

Due to their unique optical properties colloidal semiconductor quantum dots (QDs) —or nanocrystals—have proven to be valuable building blocks for many different types of applications ranging from light emitting devices ${ }^{13-15}$ to photovoltaics ${ }^{16-18}$ and sensors. ${ }^{19-21}$ The energy flow from donors to acceptors generated in FRET structures can also be optimized by the use of QDs as energy donors and/or acceptors due to their tuneable, narrow emission features. Broad absorption spectra, high photostability, and quantum yield present additional advantages for FRET applications such as light harvesting structures ${ }^{22,23}$ and sensing devices. ${ }^{19,24,25}$ The main difference between dyes, commonly used for FRET applications, and QDs is the larger size of the QDs and the inhomogeneous broadening of the QD ensemble. ${ }^{26}$ These two parameters are not taken into account by most common FRET theories, which have been developed for molecular systems and represent donors and acceptors as point dipoles. For FRET between randomly distributed, homogeneous donor and acceptor ensembles, these theories predict an acceptor concentration dependence of the FRET rate and efficiency at a fixed separation between the donor and acceptor layers, but no dependence on the donor concentration is expected. ${ }^{2,3,27}$ In order to be able to fully control and optimize FRET in QD structures the impact of inhomogeneous broadening of the QD ensemble on the FRET process has to be understood. 
Kagan et al. were the first to report on FRET in QD solids. $^{28,29}$ Since then, FRET in QD systems has been studied in combination with organic dyes in solution-based complexes $^{19,30}$ and layer structures ${ }^{31,32}$ as well as in pure QD assemblies such as clusters in solution, ${ }^{33-35}$ monodispersed QD structures, ${ }^{36-38}$ mixed donor-acceptor QD solids, ${ }^{37,39}$ mixed donor-acceptor monolayers, ${ }^{40,41}$ and donor-acceptor bilayer structures. ${ }^{36,37,42-46}$ The separated donor-acceptor bilayer structure is most interesting in terms of applications as it can be used in graded energy structures ${ }^{22,23,47}$ and it also is the geometry used in substrate-based sensing platforms (see examples in the review by Borisov and Wolfbeis ${ }^{5}$ ). So far only the distance dependence of the FRET process in the QD bilayer structure has been investigated. ${ }^{44,46}$ However, FRET rate and efficiency also depend on the acceptor concentration and additionally, the acceptor enhancement is influenced by the donor-acceptor ratio, as has already been reported for QD solids ${ }^{39}$ and monolayers. ${ }^{41}$ Furthermore, it has been shown that the donor-acceptor ratio can be used as a parameter to improve the photon efficiency of light emitting devices. ${ }^{48}$ It is important to investigate the influence of the donor and acceptor concentrations on the FRET efficiency and rate in detail to determine the effect they can have on the performance of devices, such as, for example, the sensitivity of sensors. It is particularly important to consider concentration effects in multilayer structures, such as light harvesting devices, as some of the QDs are simultaneously acting as donors and acceptors in such assemblies.

Here, we present the acceptor and donor concentration dependences of the FRET process in a donor-acceptor QD bilayer structure and highlight the influence of intra-ensemble FRET within the donor layer on the donor-acceptor inter-layer FRET. The competition between these two FRET processes gives rise to a donor concentration dependence of the FRET efficiency that is not predicted by the standard FRET theories which consider randomly distributed, homogeneous donor and acceptor ensembles. We explain how this effect can be understood within the FRET rate model as well as how to take it into account in the calculations of the FRET efficiency based on the spectral overlap and the sample geometry.

\section{THEORETICAL BACKGROUND}

Förster resonant energy transfer (FRET) is a donor deexcitation mechanism with rate $k_{\text {FRET }}$ that competes with radiative and nonradiative decay mechanisms of the excited donor state. The respective rates $k_{r}$ and $k_{n r}$ determine the intrinsic lifetime $\tau_{D}=\left(k_{n r}+k_{r}\right)^{-1}$ of the donor emission decay, which can be determined from time-resolved photoluminescence measurements, and the donor quantum yield $Q_{D}=k_{r} /\left(k_{r}+k_{n r}\right)$, which can be obtained by comparing the spectral properties of the donor with those of a wellknown luminescent reference such as Rhodamine 6G used here. As mentioned above, FRET is a strongly distance dependent process that only takes place over donor-acceptor separations $r_{D A}$ of a few $\mathrm{nm}$. The characteristic FRET distance, the Förster radius $R_{0}$, is defined as the donor-acceptor distance $r_{D A}$ at which $k_{\mathrm{FRET}}$ is as fast as the other intrinsic donor decay processes characterized by the donor decay lifetime $\tau_{D}:^{1}$

$$
k_{\mathrm{FRET}}=\tau_{D}^{-1}\left(\frac{R_{0}}{r_{D A}}\right)^{6} .
$$

The Förster radius is given by

$$
R_{0}=0.0211 \cdot\left(\frac{\kappa^{2} \cdot Q_{D}}{n^{4}} \cdot J\right)^{1 / 6}
$$

and is typically of the order of $1-10 \mathrm{~nm} .^{2,3}$ It depends on the orientation factor $\kappa^{2}$ of the donor and acceptor dipoles (which is $2 / 3$ in the case of randomly oriented dipoles, as is the case in the structures presented here), the donor quantum yield $Q_{D}$, the refractive index of the surrounding medium $n$, and the spectral overlap $J$ of the emitting donor and absorbing acceptor states. The spectral overlap

$$
J=\int_{0}^{\infty} \hat{I}_{\text {Don }}(\lambda) \cdot \varepsilon_{\text {Acc }}(\lambda) \cdot \lambda^{4} d \lambda
$$

is calculated by integrating over the area-normalized donor emission spectrum $\hat{I}_{\text {Don }}(\lambda)$ and the acceptor extinction spectrum $\varepsilon_{\text {Acc }}(\lambda) . R_{0}$ is obtained in $n m$ if the overlap $J$ is included in units of $\mathrm{nm}^{4} /(\mathrm{M} \mathrm{cm})$ in Eq. (2).

The FRET efficiency is defined as

$$
E_{\mathrm{FRET}}=\frac{k_{\mathrm{FRET}}}{\tau_{D}^{-1}+k_{\mathrm{FRET}}},
$$

and can be determined experimentally by comparing the donor lifetime in the presence of the acceptors $\tau_{D A}=$ $\left(\tau_{D}^{-1}+k_{\mathrm{FRET}}\right)^{-1}$ to the intrinsic donor lifetime $\tau_{D}$ :

$$
E_{\mathrm{FRET}}=1-\frac{\tau_{D A}}{\tau_{D}} .
$$

With Eq. (1) the FRET efficiency (4a) can also be written as

$$
E_{\mathrm{FRET}}=\frac{R_{0}^{6}}{R_{0}^{6}+r_{D A}^{6}},
$$

which allows for a calculation of the donor-acceptor distance $r_{D A}$ or simply the observation of changes in $r_{D A}$ by measuring $E_{\text {FRET }}{ }^{4}$ From Eq. (4c) it can be easily seen that the FRET efficiency is $50 \%$ for $r_{D A}=R_{0}{ }^{2,3}$

Equations (1) and (4c) describe single donor-acceptor pairs. To apply these equations to structures in which donors interact with multiple acceptors numbered $i$, the FRET rates for each acceptor at a distance $r_{i}$ from a specific donor have to be summed to obtain the FRET rate for this donor:

$$
k_{\mathrm{FRET}}=\tau_{D}^{-1} \sum_{i}\left(\frac{R_{0}}{r_{i}}\right)^{6} .
$$

Consequently Eq. (4c) modifies to

$$
E_{\mathrm{FRET}}=\frac{1}{1+\left[\sum_{i}\left(R_{0} / r_{i}\right)^{6}\right]^{-1}}
$$

in a structure where donors interact with multiple acceptors. 
Wolber and Hudson applied the rate equations of threedimensional Förster theory, summarized above, to random, two-dimensional donor-acceptor distributions. ${ }^{27}$ Furthermore, they introduced an exclusion zone $R_{e x}$ around the donors that can find application in biological systems but can also be used to take account of the QD size in mixed donoracceptor monolayers. ${ }^{41}$ This model has also been applied to the investigation of intra-ensemble FRET in monodispersed QD monolayers. ${ }^{38}$

In the bilayer structure investigated here, the acceptor layer is separated by a distance $d$ from the donor layer. Using the Wolber and Hudson model this distance can be included within $R_{e x} \cdot{ }^{27}$ Alternatively, the summation over all acceptors $i$ in Eqs. (5) and (6) can be changed into an integration over the acceptor plane by weighting with the acceptor concentration, $c_{\text {Acc }}$, as the acceptor concentration is homogenous and therefore independent of the distance $r_{i}$. The FRET rate is decreasing rapidly with distance so that the sample boundaries can be neglected and the integration can be carried out over an infinite plane:

$$
k_{\text {FRET }}=\tau_{D}^{-1} c_{\text {Acc }} \int_{0}^{\infty}\left(R_{0} / r\right)^{6} d S .
$$

Taking into account the fixed minimum distance $d$ between the donors and acceptors-in the case of QDs this is the centerto-center distance - the FRET rate can be written as

$$
k_{\mathrm{FRET}}=\frac{c_{\mathrm{Acc}} \pi R_{0}^{6}}{2 d^{4} \tau_{D}}
$$

and the expression

$$
E_{\mathrm{FRET}}=\frac{1}{1+\frac{2 d^{4}}{c_{\mathrm{Acc}} \pi R_{0}^{6}}}
$$

is obtained for the FRET efficiency in a donor-acceptor bilayer structure. It is important to note that the typical $d^{-6}$ distance dependence for a single donor-acceptor pair is replaced by a $d^{-4}$ dependence when the donor is interacting with a plane of acceptors. Additionally, it can be noted that for a random distribution of homogeneous donor and acceptor ensembles, the FRET rate and efficiency in the bilayer structure depend on the donor-acceptor separation $d$ and the acceptor concentration $c_{\text {Acc }}$, but not on the donor concentration $c_{\text {Don. }}$.

\section{EXPERIMENTAL METHODS}

Negatively charged CdTe QDs, stabilized by thioglycolic acid in aqueous solution, ${ }^{49,50}$ were used for the preparation of the FRET structures. QDs with a diameter of $2.7 \mathrm{~nm}$, and peak emission wavelength at $547 \mathrm{~nm}$, were used as donors. The acceptor QDs had a size of $3.5 \mathrm{~nm}$ and emitted at $610 \mathrm{~nm}$. The diameter of the QDs is determined from the position of the first absorption peak recorded in solution, and the concentration of the QDs in solution as well as in the monolayers were determined by the Lambert-Beer law. ${ }^{51}$

The layer-by-layer (LbL) electrostatic assembly technique s2,53 was used to prepare structures consisting of pure QD monolayers only (reference samples) and structures with donor and acceptor layers separated by a polyelectrolyte spacer.
Each polyelectrolyte bilayer consists of a negatively charged poly(sodium 4-styrene sulfonate) (PSS) and a positively charged poly(diallyldimethylammonium chloride) (PDDA) layer. Details on the preparation of the PSS and PDDA solutions (containing $0.1 \mathrm{M} \mathrm{NaCl}$ ) used in the $\mathrm{LbL}$ deposition can be found elsewhere. ${ }^{38,46}$ The PSS and PDDA polyelectrolyte bilayers were deposited by immersing the substrate alternating in the PSS and PDDA solutions for 10 min. The samples were rinsed in millipore water for $1 \mathrm{~min}$ between each deposition step and the thickness of the resulting bilayers is approximately $3 \mathrm{~nm}$, as verified using an X-ray diffraction technique.

The QD monolayers were prepared by immersion in the respective QD solution with a low QD concentration of approximately $1.5 \mu \mathrm{M}$. The concentration of the QDs in the layers was changed by varying the immersion time in the QD solution. Further information on the preparation of monodispersed QD layers with different concentrations can be found elsewhere. ${ }^{38,41}$ Samples with donor or acceptor monolayers only were prepared as reference samples. For the separated donor-acceptor layer structure, the acceptor QD layer was deposited first followed by the spacer, consisting of a varying number of PDDA and PSS bilayers and a final PDDA layer. The thickness of the spacer was varied between $0.5 \mathrm{~nm}$ (one PDDA layer only) ${ }^{42}$ and approximately $12 \mathrm{~nm}$ (four PDDA/PSS bilayers + PDDA layer). The donor QDs were deposited as the last layer.

The absorption spectra of the QD structures were recorded between 350 and $800 \mathrm{~nm}$ using a double beam UV-Vis recording spectrometer (Shimadzu UV-2401 PC). Room-temperature steady-state photoluminescence (PL) spectra were measured with a Perkin-Elmer LS 55 fluorescence spectrometer using an excitation wavelength of $400 \mathrm{~nm}$, provided by a pulsed Xenon lamp. The time-resolved PL decays of the QD layers were recorded using a PicoQuant Microtime200 time-resolved confocal microscope system with 150 ps resolution. A LDH480 laser head controlled by a PDL-800B driver (PicoQuant) provided picosecond pulses at $470 \mathrm{~nm}$ for excitation. Broad band filters centered at 500, 550, 600, and $650 \mathrm{~nm}$ were used to separate the emission from the donor and acceptor QDs, as well as to record signals from the low- and highenergy side of the QD ensemble emission for the reference samples. These filters have a full width at half maximum of approximately $(70 \pm 5 \mathrm{~nm})$. PL lifetime measurements in the donor dominated spectral range were recorded with a repetition rate of $10 \mathrm{MHz}$, whereas a lower repetition rate of $5 \mathrm{MHz}$ had to be used to record the longer decays in the spectral range dominated by the acceptor emission. The laser power was kept constant at approximately $16 \mathrm{nW}$ for all measurements. The PL decays were measured over an area of $80 \mu \mathrm{m}$ by $80 \mu \mathrm{m}$ (150 by 150 pixels) with an integration time of $4 \mathrm{~ms}$ per pixel.

\section{RESULTS AND DISCUSSION}

The optical properties of the pure donor and acceptor monolayers are important for the analysis of FRET in the donor-acceptor bilayer structure. The properties of the monolayers, including concentration effects, will be presented briefly. Then, FRET in a donor-acceptor bilayer structure 


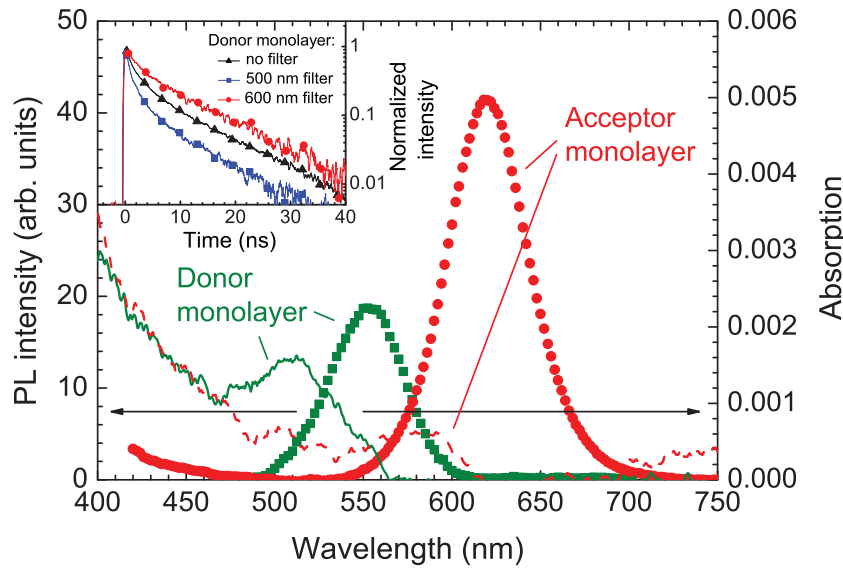

FIG. 1. (Color online) Absorption (line) and photoluminescence (PL) spectra (symbols) for donor (solid, green line, and green squares) and acceptor (dashed, red line, and red circles) QD monolayers with concentrations of $c_{\text {Don }}=1.1 \times 10^{17} \mathrm{~m}^{-2}$ and $c_{\text {Acc }}=0.28 \times$ $10^{17} \mathrm{~m}^{-2}$, respectively. In the inset the donor PL decays for the blue (blue squares) and red (red circles) side of the QD ensemble emission spectrum as well as the unfiltered decay (black triangles) are shown.

will be analyzed in detail and the acceptor and donor concentration dependences of the FRET efficiency will be discussed.

\section{A. QD monolayers}

\section{Characterization of donor and acceptor $Q D s$}

The optical properties of donor and acceptor QD monolayers are shown in Fig. 1. The first absorption maximum of the donors occurs at $510 \mathrm{~nm}$ and the PL emission is centered at approximately $553 \mathrm{~nm}$ (see Fig. 1, green solid line and squares) for a donor QD concentration $c_{\text {Don }}$ of $1.1 \times 10^{17} \mathrm{~m}^{-2}$. The PL decays of different parts of the donor ensemble emission spectrum are presented in the inset of Fig. 1(a). The initial decrease of the PL intensity $I(t)$ has been associated with holes getting trapped in defect states. ${ }^{50,54,55}$ The variation of the nonradiative decay rates $k_{n r}$ from QD to QD due to trapping gives rise to a distribution of lifetimes within the QD ensemble and consequently multi-exponential PL decays have been widely observed for CdTe QDs. Here, all PL decays were fitted by a two-exponential decay $I(t)=$ $I_{s} \exp \left(-t / \tau_{s}\right)+I_{l} \exp \left(-t / \tau_{l}\right)+I_{0}$ to take into account the non-monoexponential decay behavior of the QD ensembles. Average decay lifetimes $\tau$ are calculated as intensity-weighted means,

$$
\tau=\frac{I_{s} \tau_{s}^{2}+I_{l} \tau_{l}^{2}}{I_{s} \tau_{s}+I_{l} \tau_{l}},
$$

from the short and long lifetime component $\tau_{s}$ and $\tau_{l}$, respectively. The overall, unfiltered decay (black triangles) of the donor monolayer shown in the inset of Fig. 1 can be described by an average lifetime $\tau_{\text {noF }}=7.2 \mathrm{~ns}$. The decays on the blue (blue squares) and red side (red circles) of the donor QD ensemble emission spectrum were obtained by using a 500 and $600 \mathrm{~nm}$ broad-band filter, respectively. The average lifetime on the blue side $\tau_{\text {blue }}=4.8 \mathrm{~ns}$ is shorter than the unfiltered value $\tau_{\text {noF }}$, and the decay on the red side with a lifetime of $\tau_{\text {red }}=10.8 \mathrm{~ns}$ is longer. It has been previously shown that the optical properties of closely packed QD structures can be strongly influenced by FRET within the QD ensemble. ${ }^{38}$ For the donor QDs used in the experiments presented here a strong impact of intra-ensemble FRET on the optical properties is observed. This intra-ensemble energy transfer gives rise to the spectral redshift and is the main cause for this large difference in lifetime on the blue and red side of the QD ensemble emission observed for the QD monolayers. The concentration dependences of the optical properties arising from intra-ensemble FRET will be discussed briefly in the next section.

Similar signatures are seen in the acceptor monolayer (Fig. 1, red dashed line and circles). The acceptor absorption peak occurs at $576 \mathrm{~nm}$ (dashed line) and the PL emission peak wavelength is $619 \mathrm{~nm}$ (circles) for a monolayer with a concentration $c_{\text {Acc }}=0.28 \times 10^{17} \mathrm{~m}^{-2}$. The average acceptor PL lifetimes determined from the two-exponential fits are $\tau_{\mathrm{noF}}=12.3 \mathrm{~ns}, \tau_{\text {blue }}=6.1 \mathrm{~ns}$, and $\tau_{\text {red }}=14.6 \mathrm{~ns}$ (PL decays not shown). Similar to the donor decays, the decay on the red side is longer than the unfiltered decay and the one on the blue side is shorter.

\section{Concentration effects}

Time-resolved PL decays have been measured for monodispersed donor QD monolayers with different QD concentrations. The concentration dependence of the measured average lifetime $\tau_{\text {blue }}$ (filled squares, left-hand axis) is shown in Fig. 2. The shortening of the decay with increasing donor QD concentration is attributed to FRET from smaller to larger QDs in the inhomogeneously broadened donor ensemble. As has already been reported, ${ }^{38}$ the concentration dependence can be fitted using the theory of FRET in two dimensions developed by Wolber and Hudson. ${ }^{27}$ The full details on the application

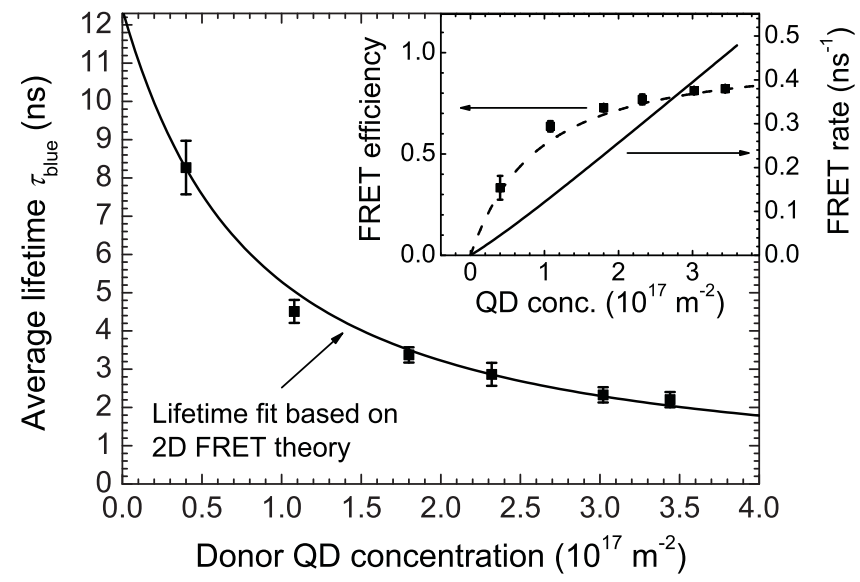

FIG. 2. Concentration dependence of the average lifetime of the PL decays on the blue side of the ensemble emission spectrum of the donor monolayers $\tau_{\text {blue }}$ (filled squares). The line represents the fit of the lifetime data using a theory of FRET in two dimensions (Ref. 27). The intra-ensemble FRET efficiency and FRET rate, calculated from the fit of the measured lifetime data, are shown in the inset. 
of this theory to monodispersed monolayers can be found in Ref. 38. The best fit of the average lifetime $\tau_{\text {blue }}$ as a function of the donor QD concentration $c_{\text {Don }}$, shown as a solid line in Fig. 2, was obtained for the fitting parameters $R_{e x}=3.0 \mathrm{~nm}$, $R_{0}=3.0 \mathrm{~nm}$, and $\tau_{\text {blue }}^{0}=12.4 \mathrm{~ns}$. $\tau_{\text {blue }}^{0}$ is the donor lifetime in the absence of FRET and corresponds to the lifetime at very low donor concentrations.

The efficiency of the intra-donor ensemble FRET process in the monodispersed donor QD monolayer can be calculated for concentration with Eq. (4b) based on the measured, concentration dependent lifetime $\tau_{\text {blue }}$ and the initial, undisturbed value $\tau_{\text {blue }}^{0}$. $E_{\text {FRET }}$ increases to $\sim 80 \%$ at high donor QD concentrations, as can be seen in the inset of Fig. 2. Also shown in the inset of Fig. 2 is the concentration dependence of the intra-ensemble FRET rate, $k_{D-D}$, obtained from the fit of the concentration dependent donor lifetime $\tau_{\text {blue }}=$ $\left(\tau_{\text {blue }}^{0-1}+k_{D-D}\right)^{-1}$.

\section{B. FRET in separated donor-acceptor layer structure}

In Fig. 3 the PL spectrum and decays of a typical donoracceptor bilayer sample are shown along with the reference spectra and decays for pure donor and acceptor monolayers at similar concentrations. The bilayer sample consists of an acceptor QD layer with $c_{\text {Acc }}=0.45 \times 10^{17} \mathrm{~m}^{-2}$, a PDDA spacer layer giving rise to a donor-acceptor center-to-center separation $r_{D A}=(3.6 \pm 0.3) \mathrm{nm}$ [based on a spacer layer thickness of $0.5 \mathrm{~nm}$ (Ref. 42) and the donor and acceptor $Q D$ radii], and a donor QD layer with $c_{\text {Don }}=1.8 \times 10^{17} \mathrm{~m}^{-2}$. The PL spectrum of the bilayer structure [filled triangles in Fig. 3(a)] shows donor emission quenching with respect to the donor reference spectrum (open squares) of $(67 \pm 5) \%$. Compared to the acceptor reference spectrum (open circles), the acceptor emission in the bilayer structure is enhanced by $(179 \pm 20) \%$, corresponding to an acceptor enhancement per donor QD of $(46 \pm 8) \%$. The acceptor enhancement per donor $\mathrm{QD}$ is calculated by weighting the acceptor PL enhancement with the donor-acceptor concentration ratio. The strong donor quenching and large acceptor enhancement show that energy is transferred efficiently from the donors to the acceptors.

In order to investigate the energy transfer mechanism more closely the time-resolved PL decays of the donors and acceptors are analyzed. The donor and acceptor PL decays have been measured on the blue and red side of the QD emission spectra, respectively, to avoid the region of spectral overlap of the donor and acceptor emission at intermediate wavelengths. In Fig. 3(b) the donor PL decays are presented for the bilayer structure (filled squares) and the donor reference (open squares). The average donor lifetime reduces from $\tau_{D}=(3.3 \pm 0.2) \mathrm{ns}$ in the reference monolayer to $\tau_{D A}=(1.7 \pm 0.1) \mathrm{ns}$ in the full structure including the acceptor QD layer. This decrease in lifetime corresponds to a FRET efficiency $E_{\mathrm{FRET}}=(48 \pm 5) \%$ as calculated with Eq. (4b). This value is very similar to the acceptor enhancement per donor QD, confirming that the additional energy received by the acceptors is transferred via the Förster mechanism and not by radiative energy transfer. Dexter energy transfer, ${ }^{56,57}$ based on an exchange mechanism, can also be excluded as a possible energy transfer process because of the relatively large donor-acceptor center-to-center
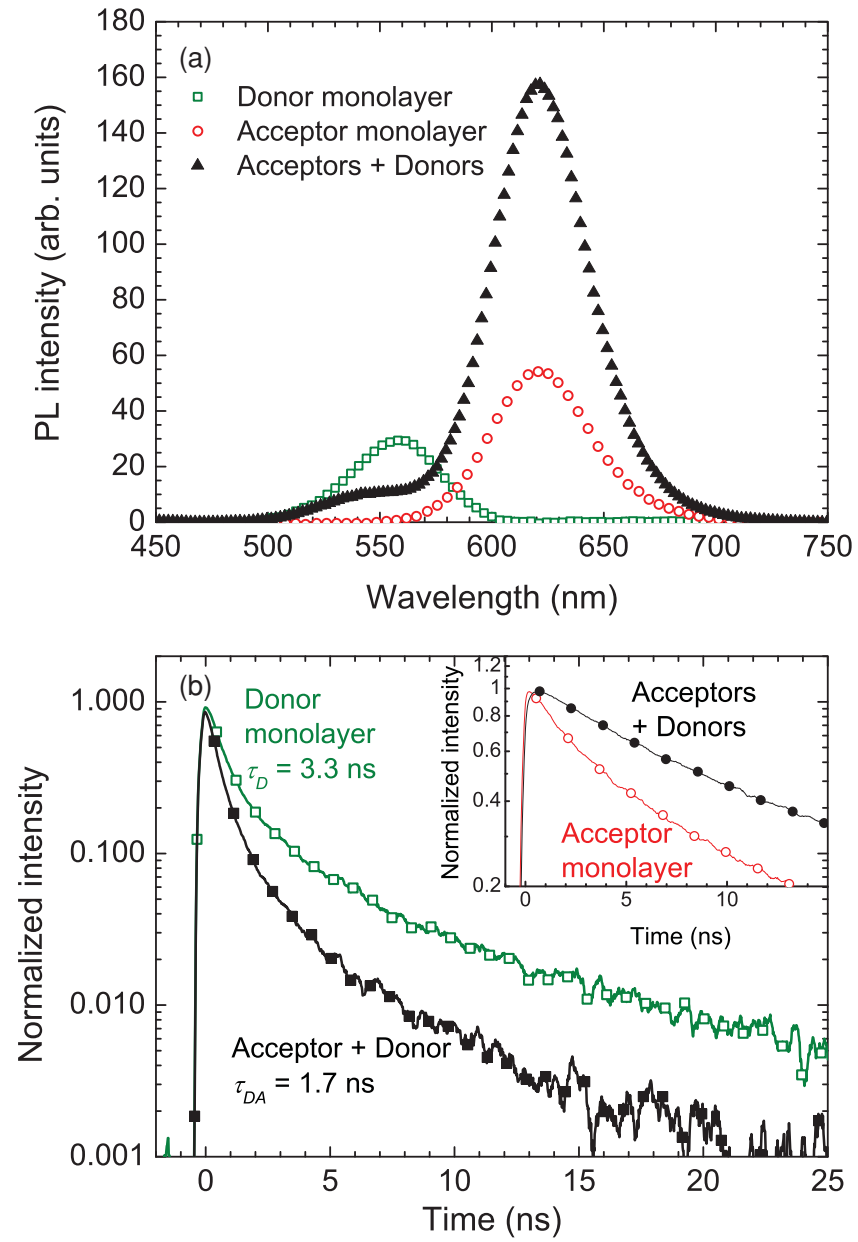

FIG. 3. (Color online) (a) Photoluminescence spectrum of a donor-acceptor bilayer structure with a donor-acceptor center-tocenter separation of $3.6 \mathrm{~nm}$ (corresponding to a layer separation of $0.5 \mathrm{~nm}$ excluding the QD radii), $c_{\text {Don }}=1.8 \times 10^{17} \mathrm{~m}^{-2}$ and $c_{\mathrm{Acc}}=$ $0.45 \times 10^{17} \mathrm{~m}^{-2}$ (filled, black triangles). The reference spectra of donor (open, green squares) and acceptor (open, red circles) monolayers are also shown. (b) The donor emission decay (measured with a broad $500 \mathrm{~nm}$ filter) in the donor-acceptor bilayer structure (filled, black squares) and in the donor reference monolayer (open, green squares). In the inset the first $12 \mathrm{~ns}$ of the acceptor emission decay (using a broad $650 \mathrm{~nm}$ filter) are shown for the donor-acceptor bilayer structure (filled, black circles) and the acceptor reference monolayer (open, red circles).

separation of $r_{D A}=(3.6 \pm 0.3) \mathrm{nm}$ in these samples. The inset in Fig. 3(b) shows the acceptor decays for the bilayer structure (filled circles) and the acceptor reference (open circles). It can be seen that the intensity maximum of the acceptor decay in the donor-acceptor bilayer structure occurs approximately $0.6 \mathrm{~ns}$ later than for the acceptor reference. This rise time is due to the additional pumping of the acceptors by the donors that occurs over a longer time than excitation by the laser pulse. In addition, due to the energy transfer, the lifetime $\tau_{A D}=(22.1 \pm 0.8) \mathrm{ns}$ of the acceptor emission decay in the bilayer structure is much longer than the PL lifetime $\tau_{A}=(14.3 \pm 0.6) \mathrm{ns}$ in the monolayer reference. 


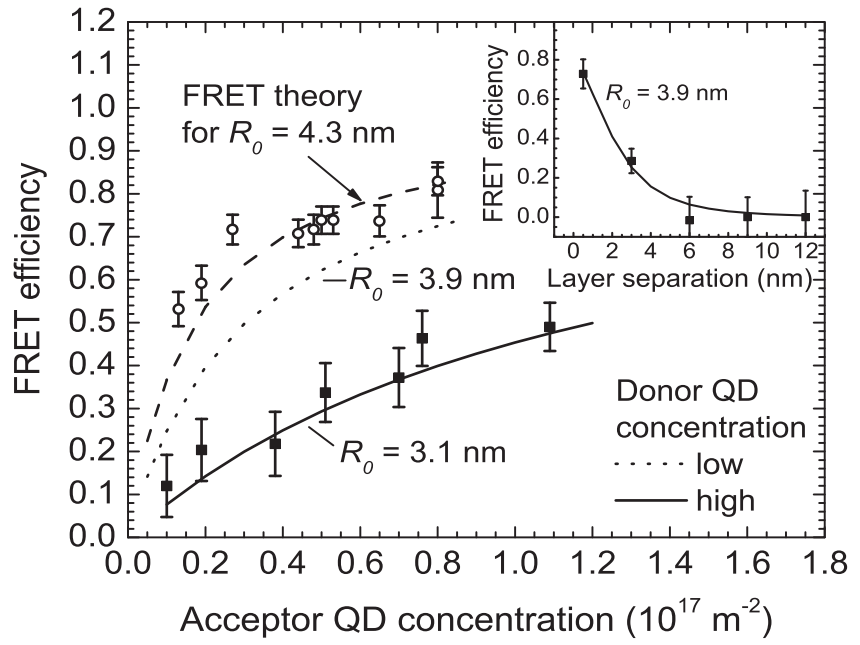

FIG. 4. Dependence of the measured FRET efficiency $E_{\mathrm{FRET}}$ on the acceptor concentration at high $\left[c_{\text {Don }}=(3.1 \pm 0.5) \times 10^{17} \mathrm{~m}^{-2}\right.$, solid squares] and low donor concentrations $\left[c_{\text {Don }}=(1.0 \pm 0.2) \times\right.$ $10^{17} \mathrm{~m}^{-2}$, open circles] for a donor-acceptor separation of $3.6 \mathrm{~nm}$. The lines represent theoretically calculated trends for $R_{0}=3.1 \mathrm{~nm}$ (solid line), $R_{0}=3.9 \mathrm{~nm}$ (dotted line), and $R_{0}=4.3 \mathrm{~nm}$ (dashed line). In the inset the distance dependence at a donor concentration of $c_{\text {Don }}=(3.9 \pm 1.2) \times 10^{17} \mathrm{~m}^{-2}$ and an acceptor concentration of $c_{\text {Acc }}=(0.85 \pm 0.10) \times 10^{17} \mathrm{~m}^{-2}$ is shown, with the theoretical trend for $R_{0}=3.9 \mathrm{~nm}$ shown as solid line.

\section{Acceptor concentration dependence}

In the inset of Fig. 4 the measured FRET efficiency (filled squares) is shown as a function of the donor-acceptor layer separation (the spacer layer thickness excluding the QD radii) for high donor concentration $c_{\text {Don }}=(3.9 \pm 1.2) \times 10^{17} \mathrm{~m}^{-2}$ and high acceptor concentration $c_{\text {Acc }}=(0.85 \pm 0.10) \times$ $10^{17} \mathrm{~m}^{-2}$. A Förster radius $R_{0}=(3.9 \pm 0.1) \mathrm{nm}$ has been calculated with Eq. (2) from the spectral overlap of the donor and acceptor reference monolayers and taking into account an average donor ensemble quantum yield of $10 \%$, as calculated from the integrated donor ensemble PL in the monolayer at the time of the measurements. An excellent agreement of the measured decrease of the FRET efficiency with increasing layer separation with theory [solid line in the inset of Fig. 4, calculated using Eq. (8)] is observed indicating the validity of the theory to describe FRET in QD systems and the value of $R_{0}$. This is in agreement with previous reports for the distance dependence of the FRET rate, ${ }^{44}$ the FRET efficiency, and acceptor enhancement ${ }^{46}$ measured in similar structures prepared by the LbL deposition technique.

The acceptor concentration dependence of the FRET efficiency with two different donor concentrations was measured for samples with a donor-acceptor separation $r_{D A}=$ $(3.6 \pm 0.3) \mathrm{nm}$ and is shown in the main graph of Fig. 4. Data for a low donor concentration $c_{\text {Don }}=(1.0 \pm 0.2) \times 10^{17} \mathrm{~m}^{-2}$ (open circles) and a higher concentration $c_{\text {Don }}=(3.1 \pm 0.5) \times$ $10^{17} \mathrm{~m}^{-2}$ (filled squares) are presented. In both cases the FRET efficiency increases with increasing acceptor concentration. For each data set $R_{0}$ is determined at the time of the measurements from the spectral overlap of the donor and acceptor reference monolayers, as discussed above. This takes account of changes in the QD quantum yield as a function of time. Considering, first, the higher donor concentration case, a value of $R_{0}=(3.1 \pm 0.1) \mathrm{nm}$ was measured from the spectral data. This is lower than that measured for the distance dependence due to a decrease in the donor quantum yield from $10 \%$ to $2.5 \%$ over time. The acceptor concentration dependence of the FRET efficiency was fitted with Eq. (8) using $R_{0}=(3.1 \pm 0.1) \mathrm{nm}$ and, as can be seen, good agreement between the experimental data and theory is achieved.

For the lower donor concentration samples the Förster radius from the spectral data was $R_{0}=(3.9 \pm 0.1) \mathrm{nm}$, as for the distance dependence samples discussed above. In this case, however, it can be seen that the absolute values for the measured FRET efficiency are higher than expected from theory and the data cannot be fitted with Eq. (8) using $R_{0}=3.9 \mathrm{~nm}$ (dotted line). Instead it is found that a value of $R_{0}=4.3 \mathrm{~nm}$ (dashed line) is required to achieve a good agreement between measured data and theoretical calculations. This discrepancy between the measured and calculated FRET efficiencies at the lower donor concentration is attributed to intra-ensemble FRET within the donor layer. The theory describing FRET between donor and acceptor planes, represented by Eqs. (7) and (8), does not take account of the inhomogeneous broadening of the ensembles, and consequently donor intra-ensemble FRET is not included. This theory describing the homogenous ensemble of donors and acceptors will be referred to as Hom.-Theory in the following sections. The impact of the donor intra-ensemble FRET on energy transfer in donoracceptor QD structures is explored in more detail in the next section. Subsequently, the acceptor concentration dependence of the FRET efficiency for a low donor concentration will be reconsidered.

\section{Donor concentration dependence}

In order to better understand the interplay between donordonor intra-ensemble FRET and donor-acceptor inter-layer FRET, the effect of the donor concentration on the donoracceptor FRET process has been investigated. The two data sets presented in Fig. 5 have been measured for bilayer structures with $r_{D A}=(3.6 \pm 0.3) \mathrm{nm}$ and acceptor QD concentrations $c_{\text {Acc }}=(0.3 \pm 0.2) \times 10^{17} \mathrm{~m}^{-2}$ (solid squares) and $c_{\text {Acc }}=(0.8 \pm 0.3) \times 10^{17} \mathrm{~m}^{-2}$ (open circles). Under these conditions, a donor concentration independent FRET efficiency of $32 \%$ (dotted line) and $73 \%$ (not included in Fig. 5 for clarity) is expected from Hom.-Theory using Eq. (8) for a donor quantum yield of $5 \%$ and $10 \%$ (corresponding to Förster radii of 3.4 and $3.9 \mathrm{~nm}$ ), respectively, for the lower and higher acceptor concentrations. However, as can be seen in Fig. 5, the FRET efficiency $E_{\text {FRET }}$ decreases with increasing donor QD concentration. The discrepancy between the measured FRET efficiency and that calculated with Hom.-Theory is largest at low donor QD concentrations, with convergence at higher concentrations. This is in agreement with what was observed for the acceptor concentration dependence discussed earlier, where agreement between Hom.-Theory and experiment was observed for the higher donor concentration.

Possible reasons for the donor concentration dependent decrease of the FRET efficiency can be considered. One reason could be the competition between donors for acceptors in the 


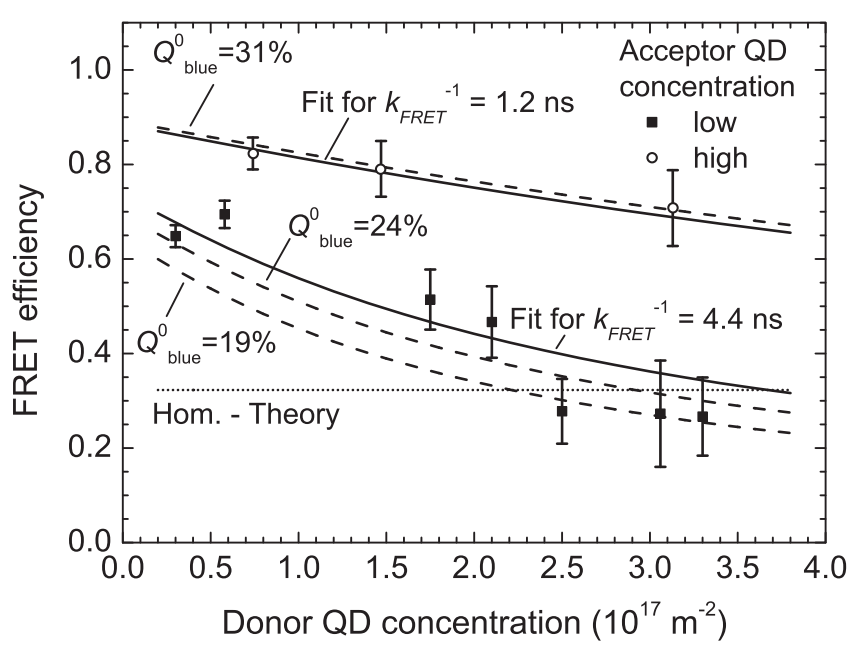

FIG. 5. Measured FRET efficiency as a function of the donor QD concentration in the bilayer structure at low $\left[c_{\text {Acc }}=(0.3 \pm 0.2) \times\right.$ $10^{17} \mathrm{~m}^{-2}$, solid squares] and high acceptor concentrations $\left[c_{\mathrm{Acc}}=\right.$ $(0.8 \pm 0.3) \times 10^{17} \mathrm{~m}^{-2}$, open circles]. Also shown is the calculated FRET efficiency expected from the FRET theory for homogenous donor ensembles with a fixed ensemble quantum yield (Hom.-Theory, dotted line), the FRET efficiency using the modified Hom.-Theory which takes into account a concentration dependent donor quantum yield (dashed line) and the fit of the experimental data with the FRET rate equation model [Eq. (4a)] including a constant FRET rate $k_{\text {FRET }}$ (solid line).

same interaction sphere (defined by twice the Förster radius) at high donor QD concentrations, but this is unlikely as not all donors are excited at the same time under the excitation conditions used in the measurements presented here. Additionally, this would also not explain why agreement between measurement and theory is observed at high concentrations. On the contrary, it would result in agreement at low concentrations with the measured FRET efficiency then decreasing to values below those predicted by theory as the donor concentration increases. The donor concentration dependence of the FRET efficiency could also originate from an efficient migration of the energy among the donors until it reaches a donor close to a suitable acceptor, when the energy would be transferred to this acceptor. This effect has, for example, been observed for chromium ions in ruby ${ }^{58}$ and leads to an increase in the FRET efficiency with increasing donor concentration. Therefore, FRET facilitated by energy migration among the donors is also not explaining the observed decrease of the FRET efficiency as a function of the donor concentration. Alternatively, the decrease of the FRET efficiency can be attributed to competition of the donor-acceptor inter-layer FRET with intra-ensemble FRET within the donor layer. This can be verified using a rate equation model. The FRET efficiency can be described by Eq. (4a), $E_{\mathrm{FRET}}=k_{\mathrm{FRET}} /\left(\tau_{D}^{-1}+k_{\mathrm{FRET}}\right)$, where $\tau_{D}^{-1}=k_{r}+k_{n r}+k_{D-D}$ is the donor decay rate in the absence of acceptors. The $k_{D-D}$ term must be included to take account of the intra-ensemble donor-donor energy transfer, discussed earlier, and consequently the donor lifetime is concentration dependent. A constant FRET rate $k_{\text {FRET }}$ is expected as the donor-acceptor FRET rate is determined by the donor-acceptor separation and the acceptor concentration.
The donor concentration does not influence the donor-acceptor inter-layer FRET rate. Using the concentration dependent lifetime data presented in Fig. 2, it is found that a constant $k_{\mathrm{FRET}}^{-1}=4.4 \mathrm{~ns}$ is sufficient to describe the donor concentration dependence of the donor-acceptor FRET efficiency in the bilayer structure (solid line in Fig. 5). This value is reasonable compared to those found in the literature for the acceptor concentration and layer separation used. ${ }^{36,37,42,44}$ As would be expected, $k_{\text {FRET }}^{-1}$ decreases for higher acceptor concentration or smaller layer separations as the donor-acceptor FRET process becomes more efficient (open circles). For an acceptor concentration of $c_{\text {Acc }}=(0.8 \pm 0.1) \times 10^{17} \mathrm{~m}^{-2}$ and $r_{D A}=$ $(3.6 \pm 0.3) \mathrm{nm}, k_{\mathrm{FRET}}^{-1}$ is reduced to approximately $1.2 \mathrm{~ns}$ (see Fig. 5). The reduction of $E_{\mathrm{FRET}}$ with increasing donor concentration is therefore not a signature for a modification of the donor-acceptor inter-layer energy transfer rate but rather the signature of competition between the donor-donor intra-ensemble FRET and donor-acceptor inter-layer FRET processes.

It is interesting to consider the origin of the divergence of the Hom.-Theory from the experimental data and how it has to be modified to take account of the inhomogeneous broadening of the donor ensemble. Contrary to experimental observations, if the concentration dependent donor lifetime measured for the monodispersed donor reference layers is included in Eqs. (7) and (8) of the Hom.-Theory, a concentration dependent FRET rate and a constant FRET efficiency are predicted (dotted line in Fig. 5), where a constant value for the ensemble quantum yield is used to calculate $R_{0}$ with Eq. (2). However, the increase of the intra-donor ensemble FRET rate with increasing donor concentration will also result in a decrease of the quantum yield on the blue side of the donor ensemble spectrum, as the quantum yield must now be expressed as $Q_{\text {blue }}=k_{r} /\left(k_{r}+k_{n r}+k_{D-D}\right)=k_{r} \tau_{D}$. This is also evidenced by a redshift of the donor emission peak with increasing donor concentration (data not shown). ${ }^{38}$ The dependence of $Q_{\text {blue }}$ on the donor concentration is the same as for the lifetime shown in Fig. 2, however, it is difficult to determine the absolute value of the quantum yield or the radiative rate for the QD sub-ensemble emitting on the blue side of the ensemble spectrum. An estimation of the value of $Q_{\text {blue }}$ at a specific donor concentration was made by considering the relative emission on the blue side and the relative absorption of the QDs emitting on the blue side with respect to the overall ensemble properties. Considering first the lower acceptor concentration data set in Fig. 5, the $Q_{\text {blue }}^{0}$ is estimated by measuring the $Q_{\text {blue }}$ from the monolayer donor reference samples. A value of $Q_{\text {blue }}=8.2$ at a donor concentration of $1.1 \times 10^{17} \mathrm{~m}^{-2}$ is estimated, which results in $R_{0}=3.7 \mathrm{~nm}$ at this donor concentration. Using the donor concentration dependence of the lifetime data in Fig. 2, this corresponds to a $Q_{\text {blue }}^{0}$ of $19 \%$. Using the same approach for the high acceptor concentration data set, $Q_{\text {blue }}=21.5 \%$ is estimated at a donor concentration of $0.6 \times 10^{17} \mathrm{~m}^{-2}$, resulting in $R_{0}=4.4 \mathrm{~nm}$. The corresponding initial quantum yield $Q_{\text {blue }}^{0}$ is $31 \%$. All estimated $Q_{\text {blue }}^{0}$ are below the quantum yield of $32 \%$ as measured in solution.

Therefore, a donor concentration dependent quantum yield has to be taken into account in the calculation of $R_{0}$ to include the effects of donor intra-ensemble energy transfer in the 
FRET Hom.-Theory. This results in a donor concentration dependent Förster radius and even though that may seem surprising at first glance, similar to the unexpected donor concentration dependence of the FRET efficiency, it is an elementary consequence of the definition of the parameter. As the FRET efficiency describes the probability for energy being transferred from a particular donor to an acceptor, it has to decrease if the probability for other donor de-excitation mechanisms increases, such as the donor intra-ensemble transfer. Similarly, as $R_{0}$ is defined as the distance at which the FRET rate is as fast as the sum of all other donor de-excitation mechanisms, $R_{0}$ will decrease with increasing donor concentration as $k_{\text {FRET }}$ is constant but the total donor decay rate $\tau_{\text {blue }}^{-1}$ increases with increasing donor concentration due to increasing donor intra-ensemble FRET (see inset of Fig. 2). Consequently, the concentration dependences of the donor lifetime $\tau_{\text {blue }}$ and $R_{0}$ in the calculation of $k_{\text {FRET }}$ with Eq. (7) cancel, and a donor concentration dependence of $E_{\mathrm{FRET}}$ is introduced in Eq. (8). The values for the FRET efficiency calculated with a modified Hom.-Theory, taking into account the estimated values $Q_{\text {blue }}\left(c_{\text {Don }}\right)$, with the same concentration dependence as for $\tau_{\text {blue }}$ shown in Fig. 2, are shown as dashed lines in Fig. 5. As pointed out above, the calculation of $Q_{\text {blue }}$ is quite difficult and therefore the error on the estimated values is in the range of $5 \%$ to $10 \%$. The trend for a few different values of $Q_{\text {blue }}^{0}$ is shown in Fig. 5. As can be seen, not only the trend but also the absolute values of the FRET efficiency can be reproduced by the modified theory which follows the same trend as the fit with the rate model (solid line). Exact agreement with the FRET rate model can be achieved for $Q_{\text {blue }}^{0}=29 \%$ for both acceptor concentrations.

In light of this discussion the acceptor concentration dependence of the FRET efficiency at two different donor concentrations, which was presented in Fig. 4, is reconsidered. Good agreement with the Hom.-Theory was obtained for the higher donor concentration, with discrepancy at the lower donor concentration where a larger $R_{0}$ had to be used to achieve a match between the measured acceptor concentration dependence of the FRET efficiency and theory. It can now be seen that the larger $R_{0}=4.3 \mathrm{~nm}$ is a consequence of the larger $Q_{\text {blue }}$ compared to the average ensemble quantum yield at low donor concentrations. When the corrected value for the quantum yield is included in the theory (dashed line in Fig. 4) good agreement with measurement is obtained.

Due to the concentration dependence of the donor reference lifetime, the FRET efficiency is not an ideal parameter to characterize the donor-acceptor FRET process in systems with high inhomogeneous broadening of the donor QD ensemble and the FRET rate is the more suitable parameter. It is important to note that if the concentration dependence of the lifetime in the monodispersed donor reference monolayers is not taken into account, but rather a constant reference lifetime is used in the calculation of the FRET efficiency, the decrease of the donor lifetime in the FRET structure could be incorrectly interpreted as an increase in FRET efficiency.

Moreover, the competition between donor intra-ensemble FRET and the donor-acceptor FRET can impair the performance of FRET devices, if the donor intra-ensemble FRET becomes dominant. This is, for example, the case for large donor-acceptor separations or low acceptor concentrations, as under these conditions the intra-donor FRET process takes place on time scales similar to or shorter than that of the donor-acceptor FRET. As shown in the inset of Fig. 2, the intra-donor FRET rate $k_{D-D}$ is of the same order of magnitude as the constant donor-acceptor FRET rate $k_{\mathrm{FRET}}$, measured for the bilayer structures presented here, and even becomes faster than $k_{\text {FRET }}$ at intermediate donor concentrations. Therefore, in any structure with closely packed donors, as could be the case for nanoclusters or QD layers and solids with high donor-acceptor ratios, these concentration effects on the FRET efficiency have to be considered if QDs with significant overlap of their emitting and absorbing states are used as FRET donors.

The modifications of the FRET theory outlined above can be adopted for all types of FRET structures, as the FRET rate model and the Förster radius are independent of the sample geometry. Only the form of Eqs. (7) and (8) and the actual concentration dependence of the donor lifetime and the quantum yield on the blue side of the donor ensemble spectrum will change.

\section{CONCLUSIONS}

We reported on Förster resonant energy transfer (FRET) in a bilayer structure consisting of separated donor and acceptor QD layers. At high donor concentrations, the distance dependence as well as the influence of the acceptor QD concentration on the FRET process agree well with the trends and values predicted by FRET theory based on homogeneous donor and acceptor ensembles. In addition, the influence of the donor QD concentration on the FRET efficiency in the bilayer structure was observed. The decrease of the FRET efficiency with increasing donor concentration does not represent a modification of the donor-acceptor FRET rate, but is a signature of the competition between donor intra-ensemble energy transfer and inter-layer donor-acceptor FRET. With increasing donor concentration it becomes likely that energy from a QD emitting on the blue side of the donor ensemble emission spectrum will be transferred to a closer energy-accepting QD available within the donor layer, instead of being transferred to a QD in the acceptor layer. The competition between these two FRET processes can be well reproduced by a rate equation model, which takes into account the concentration dependent donor lifetime due to intra-ensemble FRET in the donor reference monolayers. In addition, it was shown that by including a concentration dependent quantum yield in the FRET theory for homogeneous ensembles of donor and acceptors, the donor concentration dependence of the FRET efficiency can be reproduced as well. The concentration effects in the monodispersed donor QD layer can have an impact on the performance of QD based FRET devices and have to be taken into account when analyzing FRET in QD structures. Therefore in systems with inhomogeneously broadened donor ensembles, the FRET rate and not the FRET efficiency calculated on the basis of the donor lifetime decrease should be used to characterize the FRET process. In the QD system with a minimum donor-acceptor center-to-center separation of $3.6 \mathrm{~nm}$ a donor concentration independent donor-acceptor FRET rate as high as $(1.2 \mathrm{~ns})^{-1}$ is found, in good agreement 
with values reported in literature. Higher rates should be possible by increasing the acceptor concentration and with careful tuning of the spectral properties of the QDs to improve the spectral overlap of the donor emission and acceptor absorption, while minimizing the overlap of donor emission and absorption.

\section{ACKNOWLEDGMENTS}

We thank Robert Gunning for the X-ray diffraction measurement to determine the polyelectrolyte layer thickness. This work was financially supported by Science Foundation Ireland 05/PICA/1797.
*Author to whom correspondence should be addressed. bradlel@tcd.ie

${ }^{1}$ T. Forster, Ann. Phys. (Berlin) 2, 55 (1948).

${ }^{2}$ J. R. Lakowicz, Principles of Fluorescence Spectroscopy (Kluwer Academic/Plenum, New York, 1999).

${ }^{3}$ B. W. Van der Meer, G. Coker, and S. Y. S. Chen, Resonance Energy Transfer: Theory and Data (VCH, New York, 1994).

${ }^{4}$ L. Stryer and R. P. Haugland, Proc. Natl. Acad. Sci. USA 58, 719 (1967).

${ }^{5}$ S. M. Borisov and O. S. Wolfbeis, Chem. Rev. 108, 423 (2008).

${ }^{6} \mathrm{~J}$. W. Borst et al., Biophys. J. 95, 5399 (2008).

${ }^{7}$ A. Miyawaki, J. Llopis, R. Heim, J. M. McCaffery, J. A. Adams, M. Ikura, and R. Y. Tsien, Nature (London) 388, 882 (1997).

${ }^{8}$ P. S. Salonikidis, A. Zeug, F. Kobe, E. Ponimaskin, and D. W. Richter, Biophys. J. 95, 5412 (2008).

${ }^{9}$ D. M. Kaschak, J. T. Lean, C. C. Waraksa, G. B. Saupe, H. Usami, and T. E. Mallouk, J. Am. Chem. Soc. 121, 3435 (1999).

${ }^{10}$ D. Kuciauskas, P. A. Liddell, S. Lin, T. E. Johnson, S. J. Weghorn, J. S. Lindsey, A. L. Moore, T. A. Moore, and D. Gust, J. Am. Chem. Soc. 121, 8604 (1999).

${ }^{11}$ P. G. Van Patten, A. P. Shreve, J. S. Lindsey, and R. J. Donohoe, J. Phys. Chem. B 102, 4209 (1998).

${ }^{12}$ H. E. Song, C. Kirmaier, J. K. Schwartz, E. Hindin, L. H. Yu, D. F. Bocian, J. S. Lindsey, and D. Holten, J. Phys. Chem. B 110, 19121 (2006).

${ }^{13}$ K. S. Cho et al., Nat. Photonics 3, 341 (2009).

${ }^{14}$ V. L. Colvin, M. C. Schlamp, and A. P. Alivisatos, Nature (London) 370, 354 (1994).

${ }^{15}$ C. Bertoni, D. Gallardo, S. Dunn, N. Gaponik, and A. Eychmüller, Appl. Phys. Lett. 90, 034107 (2007).

${ }^{16}$ W. U. Huynh, J. J. Dittmer, and A. P. Alivisatos, Science 295, 2425 (2002).

${ }^{17}$ S. A. McDonald, G. Konstantatos, S. G. Zhang, P. W. Cyr, E. J. D. Klem, L. Levina, and E. H. Sargent, Nat. Mater. 4, 138 (2005).

${ }^{18}$ R. Kniprath, J. P. Rabe, J. T. McLeskey, D. Y. Wang, and S. Kirstein, Thin Solid Films 518, 295 (2009).

${ }^{19}$ I. L. Medintz, A. R. Clapp, H. Mattoussi, E. R. Goldman, B. Fisher, and J. M. Mauro, Nat. Mater. 2, 630 (2003).

${ }^{20}$ C. A. Constantine, K. M. Gattas-Asfura, S. V. Mello, G. Crespo, V. Rastogi, T. C. Cheng, J. J. DeFrank, and R. M. Leblanc, Langmuir 19, 9863 (2003).

${ }^{21}$ G. L. Wang, P. P. Yu, J. J. Xu, and H. Y. Chen, J. Phys. Chem. C 113, 11142 (2009).

${ }^{22}$ T. Franzl, T. A. Klar, S. Schietinger, A. L. Rogach, and J. Feldmann, Nano Lett. 4, 1599 (2004).

${ }^{23}$ J. Lee, A. O. Govorov, and N. A. Kotov, Nano Lett. 5, 2063 (2005).

${ }^{24}$ A. R. Clapp, I. L. Medintz, and H. Mattoussi, Chem. Phys. Chem. 7, 47 (2006).
${ }^{25}$ D. M. Willard, T. Mutschler, M. Yu, J. Jung, and A. Van Orden, Anal. Bioanal. Chem. 384, 564 (2006).

${ }^{26}$ G. D. Scholes and D. L. Andrews, Phys. Rev. B 72, 125331 (2005).

${ }^{27}$ P. K. Wolber and B. S. Hudson, Biophys. J. 28, 197 (1979).

${ }^{28}$ C. R. Kagan, C. B. Murray, and M. G. Bawendi, Phys. Rev. B 54, 8633 (1996).

${ }^{29}$ C. R. Kagan, C. B. Murray, M. Nirmal, and M. G. Bawendi, Phys. Rev. Lett. 76, 1517 (1996).

${ }^{30}$ T. Pons, I. L. Medintz, M. Sykora, and H. Mattoussi, Phys. Rev. B 73, 245302 (2006).

${ }^{31}$ E. Alphandery, L. M. Walsh, Y. Rakovich, A. L. Bradley, J. F. Donegan, and N. Gaponik, Chem. Phys. Lett. 388, 100 (2004).

${ }^{32}$ P. O. Anikeeva, C. F. Madigan, S. A. Coe-Sullivan, J. S. Steckel, M. G. Bawendi, and V. Bulovic, Chem. Phys. Lett. 424, 120 (2006).

${ }^{33}$ R. Wargnier, A. V. Baranov, V. G. Maslov, V. Stsiapura, M. Artemyev, M. Pluot, A. Sukhanova, and I. Nabiev, Nano Lett. 4, 451 (2004).

${ }^{34}$ R. Osovsky, A. Shavel, N. Gaponik, L. Amirav, A. Eychmüller, H. Weller, and E. Lifshitz, J. Phys. Chem. B 109, 20244 (2005).

${ }^{35}$ S. Mayilo, J. Hilhorst, A. S. Susha, C. Hohl, T. Franzl, T. A. Klar, A. L. Rogach, and J. Feldmann, J. Phys. Chem. C 112, 14589 (2008).

${ }^{36}$ M. Achermann, M. A. Petruska, S. A. Crooker, and V. I. Klimov, J. Phys. Chem. B 107, 13782 (2003).

${ }^{37}$ S. A. Crooker, J. A. Hollingsworth, S. Tretiak, and V. I. Klimov, Phys. Rev. Lett. 89, 186802 (2002).

${ }^{38}$ M. Lunz, A. L. Bradley, W. Y. Chen, V. A. Gerard, S. J. Byrne, Y. K. Gun'ko, V. Lesnyak, and N. Gaponik, Phys. Rev. B 81, 205316 (2010).

${ }^{39}$ S. F. Wuister, R. Koole, C. D. Donega, and A. Meijerink, J. Phys. Chem. B 109, 5504 (2005).

${ }^{40}$ C. W. Chen, C. H. Wang, Y. F. Chen, C. W. Lai, and P. T. Chou, Appl. Phys. Lett. 92, 051906 (2008).

${ }^{41}$ M. Lunz, A. L. Bradley, W. Y. Chen, and Y. K. Gun'ko, J. Phys. Chem. C 113, 3084 (2009).

${ }^{42}$ T. Franzl, D. S. Koktysh, T. A. Klar, A. L. Rogach, J. Feldmann, and N. Gaponik, Appl. Phys. Lett. 84, 2904 (2004).

${ }^{43}$ T. Franzl, A. Shavel, A. L. Rogach, N. Gaponik, T. A. Klar, A. Eychmüller, and J. Feldmann, Small 1, 392 (2005).

${ }^{44}$ D. G. Kim, S. Okahara, M. Nakayama, and Y. G. Shim, Phys. Rev. B 78, 153301 (2008).

${ }^{45}$ D. G. Kim, K. Okazaki, and M. Nakayama, Phys. Rev. B 80, 045322 (2009).

${ }^{46}$ M. Lunz, A. L. Bradley, W.-Y. Chen, and Y. K. Gun'ko, Superlattices Microstruct. 47, 98 (2010).

${ }^{47}$ A. A. Mamedov, A. Belov, M. Giersig, N. N. Mamedova, and N. A. Kotov, J. Am. Chem. Soc. 123, 7738 (2001). 
${ }^{48}$ S. Nizamoglu, O. Akin, and H. V. Demir, Appl. Phys. Lett. 94, 243107 (2009).

${ }^{49}$ S. J. Byrne, S. A. Corr, T. Y. Rakovich, Y. K. Gun'ko, Y. P. Rakovich, J. F. Donegan, S. Mitchell, and Y. Volkov, J. Mater. Chem. 16, 2896 (2006).

${ }^{50}$ A. L. Rogach, T. Franzl, T. A. Klar, J. Feldmann, N. Gaponik, V. Lesnyak, A. Shavel, A. Eychmüller, Y. P. Rakovich, and J. F. Donegan, J. Phys. Chem. C 111, 14628 (2007).

${ }^{51}$ W. W. Yu, L. H. Qu, W. Z. Guo, and X. G. Peng, Chem. Mat. 15, 2854 (2003).

${ }^{52}$ G. Decher, Science 277, 1232 (1997).
${ }^{53}$ A. Shavel, N. Gaponik, and A. Eychmüller, Eur. J. Inorg. Chem. 3613 (2005).

${ }^{54}$ S. F. Wuister, C. D. Donega, and A. Meijerink, J. Phys. Chem. B 108, 17393 (2004)

${ }^{55}$ A. M. Kapitonov, A. P. Stupak, S. V. Gaponenko, E. P. Petrov, A. L. Rogach, and A. Eychmüller, J. Phys. Chem. B 103, 10109 (1999).

${ }^{56}$ D. Dexter, J. Chem. Phys. 21, 836 (1953).

${ }^{57}$ M. Inokuti and F. Hirayama, J. Chem. Phys. 43, 1978 (1965).

${ }^{58}$ G. F. Imbusch, Phys. Rev. 153, 326 (1967). 